Article

\title{
Systematic Performance Comparison of (Duobinary)-PAM-2, 4 Signaling under Light and Strong Opto-Electronic Bandwidth Conditions
}

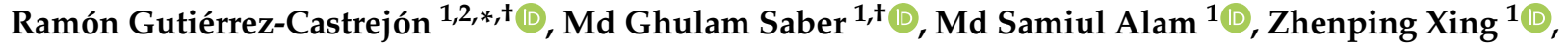 \\ Eslam El-Fiky 1,3(-), Daniel E. Ceballos-Herrera ${ }^{2}{ }^{-0}$, Fabio Cavaliere ${ }^{4}{ }^{-}$, Gemma Vall-Llosera ${ }^{5}$, Luca Giorgi ${ }^{4}$, \\ Stephane Lessard ${ }^{6}$, Robert Brunner ${ }^{6}$ and David V. Plant ${ }^{1}$
}

1 Department of Electrical and Computer Engineering, McGill University, Montreal, QC H3A 0E9, Canada; md.saber@mail.mcgill.ca (M.G.S.); md.samiul.alam@mail.mcgill.ca (M.S.A.);

zhenping.xing@mail.mcgill.ca (Z.X.); eslam.elfiky@mail.mcgill.ca (E.E.-F.); david.plant@mcgill.ca (D.V.P.)

2 Institute of Engineering, Universidad Nacional Autónoma de México UNAM, Cd. Universitaria, 04510 Mexico City, Mexico; DCeballosH@iingen.unam.mx

3 Department of Electrical Engineering, Alexandria University, Alexandria 21544, Egypt

4 Ericsson, Via Giuseppe Moruzzi, 1, 56124 Pisa, Italy; fabio.cavaliere@ericsson.com (F.C.); luca.giorgi@ericsson.com (L.G.)

5 Ericsson Research, Ericsson AB, 16480 Stockholm, Sweden; gemma.vall-llosera@ericsson.com

6 Ericsson, 8275 Trans-Canada Route, Saint-Laurent, Montreal, QC H4S 0B6, Canada; stephane.lessard@ericsson.com (S.L.); robert.brunner@ericsson.com (R.B.)

check for

updates

Citation: Gutiérrez-Castrejón, R.; Saber, M.G.; Alam, M.S.; Xing, Z.; El-Fiky, E.; Ceballos-Herrera, D.E.; Cavaliere, F.; Vall-Llosera, G.; Giorgi, L.; Lessard, S.; et al. Systematic Performance Comparison of (Duobinary)-PAM-2,4 Signaling under Light and Strong

Opto-Electronic Bandwidth

Conditions. Photonics 2021, 8, 81.

https://doi.org/10.3390/photonics 8030081

Received: 19 February 2021

Accepted: 16 March 2021

Published: 18 March 2021

Publisher's Note: MDPI stays neutral with regard to jurisdictional claims in published maps and institutional affiliations.

Copyright: (c) 2021 by the authors. Licensee MDPI, Basel, Switzerland. This article is an open access article distributed under the terms and conditions of the Creative Commons Attribution (CC BY) license (https:/ / creativecommons.org/licenses/by/ $4.0 /)$.
* Correspondence: RGutierrezC@iingen.unam.mx; Tel.: +52-55-5623-3600 (ext. 8824)

+ Contributed equally to this work.

\begin{abstract}
We present a systematic comparison of PAM-2 (NRZ), Duobinary-PAM-2, PAM-4, and Duobinary-PAM-4 (duo-quaternary) signaling in the context of short-reach photonic communications systems using a Mach-Zehnder modulator as transmitter. The effect on system performance with a relaxed and constrained system's opto-electronic bandwidth is analyzed for bit rates ranging from 20 to $116 \mathrm{~Gb} / \mathrm{s}$. In contrast to previous analyses, our approach employs the same experimental and simulation conditions for all modulation formats. Consequently, we were able to confidently determine the performance limits of each format for particular values of bit rate, system bandwidth, transmitter chirp, and fiber dispersion. We demonstrate that Duobinary-PAM-4 is a good signaling choice only for bandwidth-limited systems operating at relatively high speed. Otherwise, PAM-4 represents a more sensible choice. Moreover, our analysis put forward the existence of transition points: specific bit rate values where the BER versus bit rate curves for two different formats cross each other. They indicate the bit rate values where, for specific system conditions, switching from one modulation to another guarantees optimum performance. Their existence naturally led to the proposal of a format-selective transceiver, a component that, according to network conditions, operates with the most adequate modulation format. Since all analyzed modulations share similar implementation details, signaling switching is achieved by simply changing the sampling point and threshold count at the receiver, bringing flexibility to IM/DD-based optical networks.
\end{abstract}

Keywords: amplitude modulation; filtering; optical receivers; partial response signaling

\section{Introduction}

While we are approaching the day when coherent technology will become a sufficiently cost- and power-efficient alternative, transmission based on intensity-modulation with direct-detection (IM/DD) will continue to predominate in short-reach transmission systems. Although there is a wide range of currently available IM/DD formats [1,2], the simplest and most well-known, pulse-amplitude modulation of two levels (PAM-2) and its extension to four levels, termed quaternary pulse-amplitude modulation (PAM-4) [3-5], 
are still the formats of choice for the implementation of optical fiber transmission systems according to worldwide-accepted standards, recommendations, and multi-source agreements. Particularly relevant references are Ethernet standards such as $100 \mathrm{GbE}$ and 400 GbE [6], passive optical network (PON) conventions such as XG-PON1, 10 G and 25 G EPONs [7], and possibly, near future silicon-photonics-based X-haul interconnects for 5G architectures using IM/DD [8]. A closely related modulation format, attractive because of its narrow bandwidth (BW) and tolerance to fiber chromatic dispersion (CD), is the so-called electrical duobinary (DB) format, the most prominent representative of partial response formats [9]. Although the term normally refers to a three-level signal produced from PAM-2 through spectral shaping, there is a PAM-M equivalent, here referred to as DB-PAM-M, whose optical implementation for $M=4$ has recently received attention from the industry and academia [10-15]. Formally known as duo-quaternary modulation, the case of $M=4$ is interesting because, despite being a seven-level signal, it shares with its $M=2$ counterpart the appealing characteristics of tolerance to $\mathrm{CD}$, relatively narrow BW, acceptable receiver sensitivity performance, and reasonably simple implementation [16]. Since it is derived from a PAM-4 sequence, it has the advantage of transporting two bits per symbol, increasing its spectral efficiency by a factor of two as compared to DB-PAM-2. Although DB-PAM-4 emerged more than 50 years ago [17], three factors explain the recent interest in this format. First, the adoption by the telecommunications industry of PAM-4 as the most commonly accepted format for the implementation of high-speed IM/DD photonic systems. Second, the need to transmit such high data rates employing a physical layer infrastructure (either already installed or based on off-the-shelf components) originally conceived for less demanding environments; that is, infrastructure based on the use of transceivers that normally exhibit comparatively lower opto-electronic BW. Third, the convenience of using a more CD-tolerant format to preserve the system's reach when upgrading the bit rate. As a result of this interest, there have been reports mainly aiming to augment the value proposition of DB-PAM-4 and to explore its performance limits $[18,19]$. The investigations that compare PAM-4 and DB-PAM-4 are also interesting [13-15,20-25]. For instance, in [22], the better performance of PAM-4 over DB-PAM-4 is demonstrated at $18 \mathrm{~Gb} / \mathrm{s}$ using a directly-modulated laser (DML) and a PIN photodiode, both exhibiting a BW of $10 \mathrm{GHz}$. However, according to [24], it is DB-PAM-4 which performs better under back-to-back (BtB) conditions at $112 \mathrm{~Gb} / \mathrm{s}$. After $10 \mathrm{~km}$ transmission over standard single-mode fiber (SSMF), no performance difference between formats was observed [24]. Similarly to other studies $[10,13]$, these measurements were carried out at a single optoelectronic BW (electro-absorption modulator with $27 \mathrm{GHz}$ BW and a PIN-TIA combination having $35 \mathrm{GHz} \mathrm{BW})$ and bit rate $(112 \mathrm{~Gb} / \mathrm{s})$, and therefore the effect of varying these system parameters on the performance of each modulation format was not systematically investigated. In the context of PONs, Torres-Ferrera et al. [25] analyzed the impact on power budget by varying the system BW when a $50 \mathrm{Gbd}$ signal is employed. It was shown that DB-PAM-4 outperforms PAM-4 in BW-constrained systems, whereas the opposite occurs under sub-optimal filtering conditions. Following these achievements, it is clear that a thorough and systematic analysis along these lines is welcome. Such a study would be useful to decide which is the most adequate IM/DD format, from a system designer perspective, to be employed under certain conditions of bit rate, system BW, transmitter chirp, and fiber dispersion. The purpose of the present contribution is to fill this gap, thus complementing previous analyses. The main finding of our novel approach is that, given certain conditions of opto-electronic BW, we can produce BER versus bit rate curves for different modulation formats that exhibit the characteristic of crossing each other. We have dubbed these intersections "transition points." Since normally the functional relation between BER and bit rate in an optical system is not constant, the existence of these transition points is important because they enable the possibility of keeping the transmission system within certain performance limits as the bit rate is varied. This can be accomplished by simply changing the employed modulation format. In other words, our analysis put forward the fact that one convenient way to extend the bit rate range on which a system 
with particular BW conditions can operate is to change its modulation format, choosing among PAM-2, DB-PAM-2, PAM-4, and DB-PAM-4. Of course, the analysis can be extended to other values of $M$ [26]. We must bear in mind, notwithstanding, that higher-order modulation formats become less tolerant to signal-to-noise ratio (SNR) degradation [27].

The existence of the aforementioned transition points for the analyzed modulation formats naturally leads to the proposal of a format-selective transceiver, a component that, according to network conditions, operates with the most adequate modulation format to maintain a certain performance level. The optimum points to switch from one format to another are dictated by the transition points, which can be determined by experiments or simulations. Indeed, the transition points indicate the bit rate values where, for specific system conditions, switching from one modulation format to another guarantees optimum performance. The proposed transceiver then works similarly as a gearbox, changing gears (formats) to allow the increase in system speed. Although the idea of a multi-bit rate and multi-format transceiver is not entirely new [27-29], our proposal is, to the best of our knowledge, the first one of its kind to use PAM-M modulation formats together with their DB counterparts to set-up a flexible transceiver. This choice of signaling is especially attractive because a change in modulation format can be easily implemented, as discussed below, and hence the hypothetical transceiver can form part of an integrated opto-electronic module, ideally exhibiting low complexity and power consumption. Previous transceiver proposals and designs instead aim to bring flexibility to coherent optical networks using two-dimensional [30] and more sophisticated multi-dimensional modulation formats in combination with constellation shaping [31]. They have also employed multi-carrier intensity modulation (e.g., DMT) to perform optimum dynamic spectrum allocation within a certain granularity limit [32]. The IM/DD approach followed here is simpler, but still effective, as discussed below.

In summary, in this article, we first present an analysis of system performance as a function of bit rate for (DB)-PAM-2,4 in the context of short-reach optical transmission systems operating in O-band. This transmission window is increasing in popularity among different standardization bodies, e.g., IEEE P802.3ca 50G EPON [33] and IEEE P802.3bs $400 \mathrm{~Gb} / \mathrm{s}$ Ethernet [6], and we envision that the 100G EPON will use the O-band as well. Two different system BW scenarios, consisting of light and strong filtering, are experimentally addressed, and the effect of $\mathrm{CD}$ is examined. Our approach demonstrates that the choice of optimal modulation format depends on the specific conditions of bit rate and system BW. Therefore, our results not only represent a valuable guide in the selection of the most appropriate modulation format for a particular system deployment, but uncovers the convenience of employing a format-selective element to switch the initially chosen modulation format to a different one if the prevailing system conditions, such as bit rate or reach, eventually change. This paper is divided into seven main sections. Section 2 reviews precoding and detection of partial response signals. Basically, a tutorial on the theoretical framework that supports our work and serves as a reference for the discussions presented within this paper. Section 3 describes the experimental and simulation details. Section 4 serves as the motivation for our study. It discusses the evolution of a signal in a BW-constrained environment as the bit rate increases, pointing out the relevance of partial response signaling. Section 5 is devoted to conveying the main results of our investigation in BtB and fiber transmission contexts, whereas Section 6 deals with the technical details of the hypothetical format-selective transceiver based in (DB)-PAM modulation. This is followed by the conclusions, presented in Section 7.

\section{Detection of Duobinary Signals}

Let a PAM-M data stream of $n$ symbols, $\left[a_{n}\right] \in\{0,1, \ldots, M-1\}$, be encoded as a PAM-M signal, where $M=2,4$. Due to the finite BW exhibited by all the components of a transmission system, the eye diagram of the received signal under favorable transmission conditions will look like the one shown in Figure 1a (although a PAM-4 eye diagram is displayed, the following discussion also holds for $M=2$ ). Thanks to the simplicity of 
$\mathrm{IM} / \mathrm{DD}$, the received version of $\left[a_{n}\right],\left[a_{n}^{\prime}\right]$, can, in principle, be recovered by sampling the directly detected signal at sampling point $\mathrm{A}$, followed by conventional slicing via thresholding. An alternative approach to recovering the data stream $\left[a_{n}^{\prime}\right]$, nonetheless, consists of sampling the signal at point $\mathrm{B}$ and then slicing using $(2 M-2)$ thresholds, leading to a $(2 M-1)$ levels sequence; the so-called DB sequence [17]. [ $\left.a_{n}^{\prime}\right]$ is then obtained by applying modulus $M$ operation. The use of the alternative approach is particularly interesting when a high-speed PAM-M signal is transmitted through a communication system made up of cost-effective, narrow opto-electronic BW components, leading to an eye diagram that looks like the one shown in Figure $1 \mathrm{~b}($ for $M=4)$. Clearly, due to the strong filtering effect, slicing at the (2M-1) levels sampling point (B) will result in better identification of the correct data stream elements as compared to slicing at the traditional $M$ levels sampling point (A), where the limited channel BW has a markedly reduced eyeopening. In other words, in this case, recovering the DB-PAM-M signal will result in a better BER than recovering the plain PAM-M signal. In the following sections, we will experimentally show to what extent this statement is correct.

a)

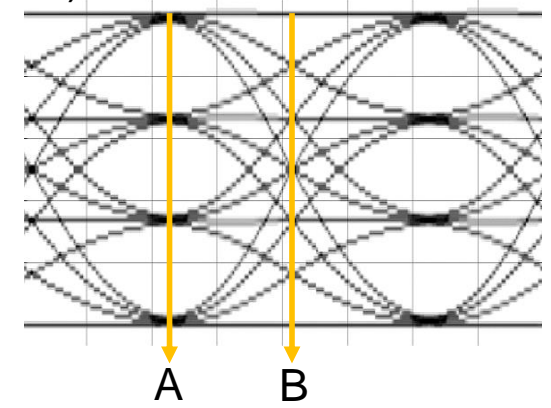

b)

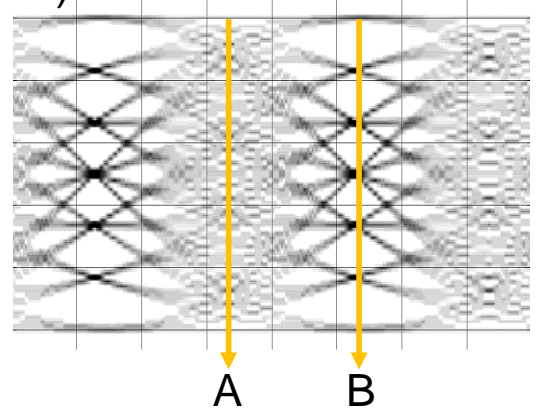

Figure 1. Eye diagrams of PAM-4 signal under light (a) and strong (b) filtering effect. A and $\mathrm{B}$ represent the sampling points used to retrieve the PAM-4 and the 7-level DB-PAM-4 signals, respectively.

The formalism behind the DB approach $[2,12,16,17]$ is based on approximating the filtering effect of the overall system (including any optical filter) through a one-tap addand-delay filter acting upon a PAM-M transmitted data stream, $\left[b_{n}\right] \in\{0,1, \ldots, M-1\}$. The filter equation can be cast as

$$
c_{k}=b_{k}+b_{k-1}, k=1,2,3, \ldots, n ；
$$

where sequence $\left[c_{n}\right] \in\{0,1, \ldots, 2 M-2\}$ becomes the digital counterpart of the $(2 M-1)$ levels sampled analog signal. Assuming $\left[a_{n}\right]=\left[a_{n}^{\prime}\right]$ (ideal case of no errors), then $\left[a_{n}\right]$ can be simply recovered using

$$
a_{k}=\left(c_{k}\right) \bmod M .
$$

Note, however, that for this expression to be true, $\left[b_{n}\right]$ must fulfill the following condition

$$
b_{k}=\left(a_{k}-b_{k-1}\right) \bmod M .
$$

Operation (3) is called precoding [17], whereas $\left[c_{n}\right]$ represents the DB-PAM-M sequence. The need for precoding in DB transmission can be demonstrated by noting from Equations (1) and (2) that since $a_{k}=\left(b_{k}+b_{k-1}\right) \bmod M$, then (3) can be written as $b_{k}=\left(\left(b_{k}+b_{k-1}\right) \bmod M-b_{k-1}\right) \bmod M$. Equality follows from employing the associative property of $\bmod M$ operation and the fact that $b_{k}=\left(b_{k}\right) \bmod M$.

The advantage in terms of spectral efficiency exhibited by DB-PAM- 4 over PAM- 2 is the result of following two different approaches. Both are graphically depicted in Figure 2. On one hand, a gain in spectral efficiency is achieved by increasing the cardinality of the amplitude modulation to higher values of $M$, that is, increasing the number of bits 
associated with each symbol (or amplitude level) of the PAM-M alphabet. For instance, in PAM-8, each of the eight symbols carries three bits. This approach corresponds to the upper path in the diagram of Figure 2. On the other hand, the signal BW can be reduced for a certain transmission rate by extending the duobinary concept to polybinary modulation. This is the approach followed in the lower path of Figure 2. It consists of using heavier spectral shaping.

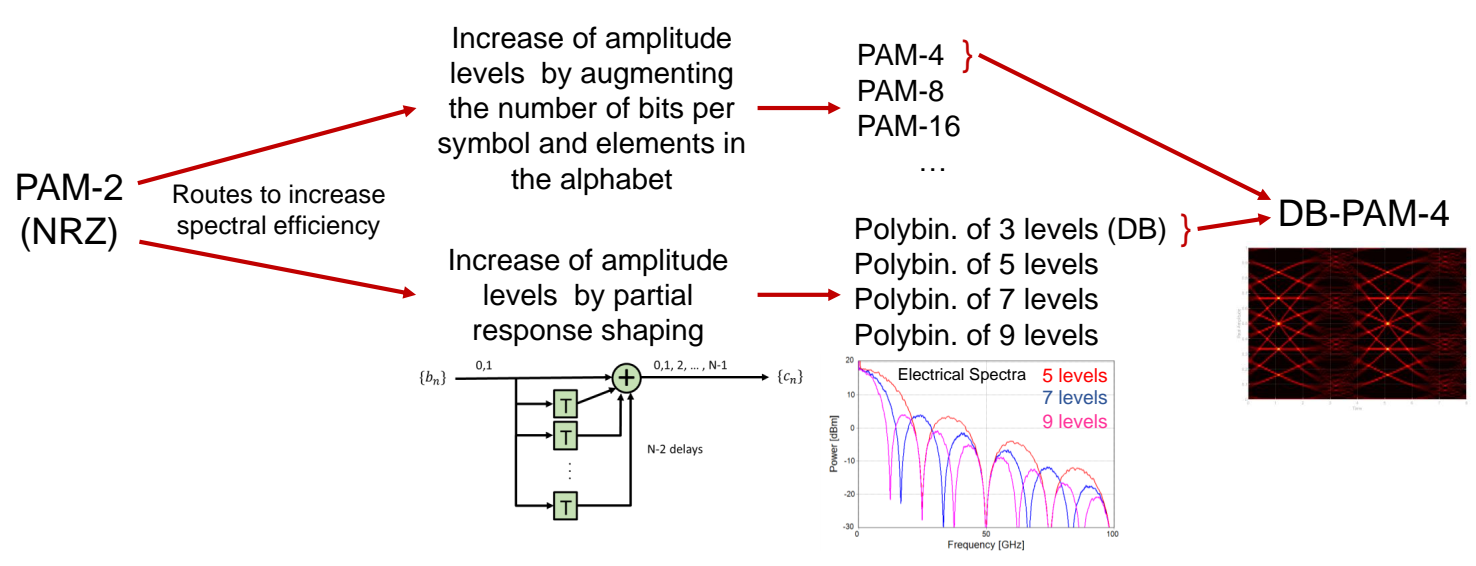

Figure 2. Diagram of two paths that can be followed to increase spectral efficiency in IM/DD systems. Their combination results in DB-PAM-4 modulation and, eventually, polybinary PAM-M modulation.

Although the increase in spectral efficiency associated with higher values of $M$ in optical PAM-M modulation is well-known [34,35], polybinary modulation has received less attention. A polybinary signal of $N$ levels is produced by using an add-and-delay filter consisting of $N-2$ delays (see diagram in Figure 2) [16]. The corresponding equation reads

$$
c_{k}=b_{k}+b_{k-1}+b_{k-2}+\cdots+b_{k-(N-2)}, k=1,2,3, \ldots, n .
$$

Equation (1) then becomes a particular case of Equation (4) when $N=3$. Precoding, in this case, is necessary to prevent error propagation. The corresponding equation is [16]

$$
b_{k}=a_{k} \otimes b_{k-1} \otimes b_{k-2} \otimes \cdots \otimes b_{k-(N-2)} ;
$$

where $\otimes$ stands for XOR operation. Note that Equation (5) reduces to (3) when $N=3$ and $M=2$. An appealing property of polybinary signaling is that the marks and spaces pertaining to $\left[a_{n}\right]$ uniquely correspond to the even and odd-numbered levels of $\left[c_{n}\right]$, respectively [17]. This simplifies the decoding process, since $\left[a_{n}\right]$ can be recovered from $\left[c_{n}\right]$ by means of $a_{k}=\left(c_{k}\right) \bmod 2$. This can be accomplished because although a polybinary signal has $N$ levels when represented in the time-domain, it still runs at the speed of a plain NRZ signal since each level carries only one bit (instead of a multi-bit symbol). Hence, the bit period in polybinary and NRZ signals is the same.

According to Figure 2, DB-PAM-4 is then the result of combining an increase in cardinality (to $M=4$ ), together with enough filtering to achieve polybinary modulation (with $N=3$ ). It hence exhibits a narrower BW than either a PAM-4 signal or a DB signal, all transmitting at the same bit rate. This is confirmed by comparing the simulated $100 \mathrm{~Gb} / \mathrm{s}$ electrical spectra shown in Figure 3. In bandwidth-unconstrained scenarios, the transmission of $M$-levels PAM-M signals should generally be preferred over its DB counterpart due to their lower number of amplitude levels and because this format is more tolerant to SNR degradation than DB-PAM-M [36]. However, $(2 M-1)$ levels DB-PAM-M signals may show better performance when transmitted over a BW-limited system. As shown below, the adequate choice of modulation format mainly depends on system bandwidth and bit rate. 


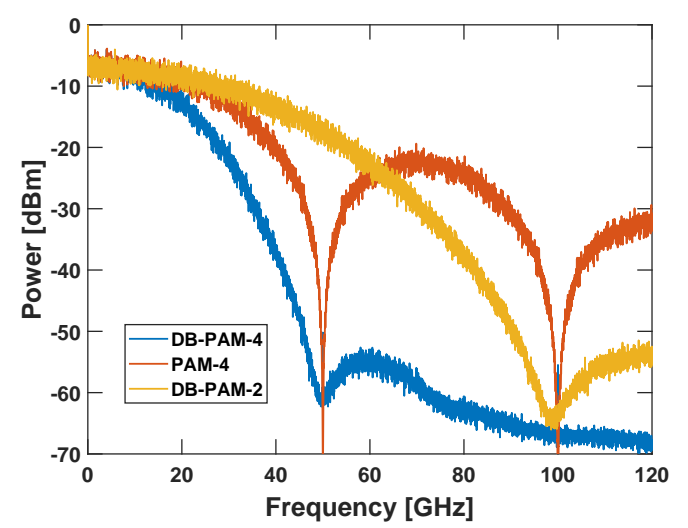

Figure 3. Simulated electrical spectra of noiseless $100 \mathrm{~Gb}$ /s signals encoded with DB-PAM-4, PAM-4 and DB modulation formats.

In this work, we will focus on the analysis of PAM-2, DB-PAM-2, PAM-4, and DBPAM-4 modulation formats. Therefore, a practical approach to carry out the investigation with minimal modifications at the transmitter end consists of using Equation (3) to transmit a pre-coded data stream $\left[b_{n}\right]$. If the system BW is ample enough and the $M$-levels of the PAM-M signal can be distinguished in the corresponding eye diagram at the receiver, then sample at point A (see Figure 1a), slice the signal with $M-1$ thresholds and apply Equations (1) to undo precoding and (2) to retrieve the original transmitted sequence. In contrast, if the system BW is rather narrow (with respect to the bit rate), sample at point B (see Figure $1 \mathrm{~b}$ ) and slice the signal with $2 M-2$ thresholds to "naturally" obtain the DB-PAM-M sequence $\left[c_{n}\right]$. Then, use Equation (2) to recover the original sequence. In the subsequent sections, we have followed this convenient approach.

\section{Experimental Setup}

Figure 4 presents the block diagram of our setup, which is designed to carry out transmission using PAM-2,4 and DB-PAM-2,4 modulation and operation at bit rates ranging from 20 to $116 \mathrm{~Gb} / \mathrm{s}$ (depending on the selected modulation format, as shown below). The off-line generated PAM-M sequence is initially precoded following Equation (3) and converted into an analog electrical signal using an 8-bit digital-to-analog converter (DAC) operating at $64 \mathrm{GSa} / \mathrm{s}$. The DAC is compensated with pre-emphasis, but no pulse shaping was applied. The DAC output is connected to an RF amplifier (SHF 806E) which then drives a silicon Mach-Zehnder modulator (MZM) with $20 \mathrm{GHz}$ 3-dB opto-electronic BW [5], operated at the quadrature point. An optically modulated signal with an approximate outer extinction ratio (ratio between the lowest and highest levels) of $4 \mathrm{~dB}$ is then generated. It is, in principle, a replica of the electrical PAM-M signal. A distributed feedback (DFB) laser emitting at $1309.7 \mathrm{~nm}$ is used as the CW source. This wavelength is $8 \mathrm{~nm}$ blue-shifted from the zero-dispersion wavelength (ZDW) of the 10-km SSMF that is used for data propagation. The back-to-back (BtB) condition has been analyzed as well. A variable optical attenuator (VOA) is employed in conjunction with a power meter monitor to ensure the injection of a signal into the optical front-end (OFE) with, in all cases, an average power of about $-4 \mathrm{dBm}$. The OFE is an optical receiver that consists of a PIN photodiode and a linear trans-impedance amplifier (TIA) from MACOM, exhibiting a combined BW of $40 \mathrm{GHz}$. An Agilent real-time oscilloscope (RTO) with a maximum sampling rate of $80 \mathrm{GSa} / \mathrm{s}$ served as analog-to-digital converter (ADC). Two system bandwidths, mainly determined by the RTO, were investigated: a low BW case where the effective receiver $\mathrm{BW}$ was set to $11 \mathrm{GHz}$ (with a "brick-wall" profile), and a high-BW case where the effective receiver BW was set to $23 \mathrm{GHz}$ (with a "brick-wall" profile). Although slightly more relaxed, the former (latter) BW conditions resemble those found in $10 \mathrm{G}(25 \mathrm{G})$ short-reach transmission systems. The off-line processing at the receiver, performed using Matlab, consisted of a simple linear feed-forward equalizer (FFE) with 11 taps for the low-BW case and 131 taps for the high-BW 
case. For ease of comparison, the same equalization conditions prevailed irrespective of the employed bit rate and modulation format. For each of the two system BWs, two different digital sequences were derived from the received signal. On one hand, we sampled the signal at point A (see Figure 1) and conducted slicing with $M-1$ thresholds to obtain a PAM-M sequence. Then, Equations (1) and (2) were applied to recover the originally launched PAM-M symbols stream. On the other hand, we sampled the signal at point $B$ (see Figure 1) and conducted slicing with $2 M-2$ thresholds to recover the DB-PAM-M sequence. In this case, only Equation (2) was necessary to recover the originally launched PAM-M symbols stream. After straightforward PAM-M decoding, the bit-error-ratio (BER) was calculated by comparing the received and the transmitted bit sequences.

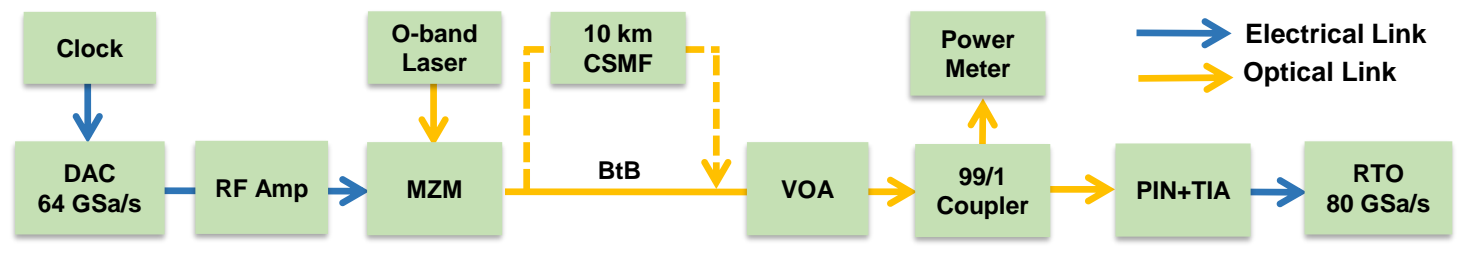

Figure 4. Experimental setup. Precoded PAM-M signals at different bit rates are launched and retrieved as PAM-M or DB-PAM-M sequences after being captured by the RTO. $M=2,4$. Adequate sampling and slicing using, respectively, $M-1$ and $2 M-2$ thresholds, are required.

The simulation work that complemented the experimental analysis was carried out in a co-simulation Matlab/OptiSystem platform [37]. Matlab processed electrical and digital signals, while the OptiSystem simulated transmission in the optical domain, thus emulating the actual experimental setup. We employed standard optical component models and neither FFE equalization nor DAC/ADC limitations were included in the simulations. We took care to consider all $\mathrm{BW}$ constraints through adequate low-pass filtering of the transmitted signal. For the MZM, we set a $\mathrm{V}_{\pi}$ of $6 \mathrm{~V}$ with a bias voltage of $4.5 \mathrm{~V}$ at the quadrature point and a 3-dB opto-electronic BW, simulated through a 5th-order Bessel filter, of $20 \mathrm{GHz}$. We employed a single-polarization, standard split-step dispersive linear fiber model with an attenuation of $0.35 \mathrm{~dB} / \mathrm{km}$. Finally, for the OFE, we selected a PIN photodiode that included thermal and shot noise modeling via Gaussian distributions, followed by a TIA. The overall BW of the OFE was set to $40 \mathrm{GHz}$. Note, however, that the actual receiver BW is determined by the RTO, where cascaded 4th-order Bessel and brick-wall low-pass filters (LPF) were utilized. The simulation parameters of the receiver are presented in Table 1.

Table 1. Main parameters of the receiver used in the simulations.

\begin{tabular}{lcc}
\hline Parameter & Value & Units \\
\hline Responsivity & 0.7 & $\mathrm{~A} / \mathrm{W}$ \\
TIA conversion gain & 1400 & $\Omega$ \\
Input current noise density & 18 & $\mathrm{pA} / \sqrt{\mathrm{Hz}}$ \\
\hline RTO Low-bandwidth case & & \\
4th-Order Bessel LPF bandwidth & 7.3 & $\mathrm{GHz}$ \\
Brick-wall LPF bandwidth & 11 & $\mathrm{GHz}$ \\
\hline RTO High-bandwidth case & & $\mathrm{GHz}$ \\
4th-Order Bessel LPF bandwidth & 15.3 & $\mathrm{GHz}$ \\
Brick-wall LPF bandwidth & 23 & \\
\hline
\end{tabular}

\section{Effect of System Bandwidth}

The evolution of the received four-level signal as the transmitted bit rate increases in a BtB system with a constant BW, is presented using simulated electrical eye diagrams in Figure 5. As the bit rate increases, the lowpass filtering effect produced by the BW of the system components becomes more severe, leading to an increase in eye closure 
at the "traditional" sampling point (A as defined in Figure 1). At $80 \mathrm{~Gb} / \mathrm{s}$, the signal levels cannot be distinguished anymore without the aid of adequate signal processing (e.g., FFE). At $100 \mathrm{~Gb} / \mathrm{s}$, the three eyes are completely closed. In contrast, the eyes that are centered at the alternative sampling point (B according to Figure 1$)$, which corresponds to DB transmission, remain comparatively unaffected as the bit rate increases. Even at $100 \mathrm{~Gb} / \mathrm{s}$, the seven levels of the DB-PAM-4 signal can be distinguished. At $120 \mathrm{~Gb} / \mathrm{s}$ no symbols can be extracted at either sampling point because the distortions produced by the relatively narrow filters are too strong. A similar situation occurs with a 2-level signal. However, since the overall BW of these signals is higher, the distortions manifest themselves at lower bit rates. From these remarks, it is straightforward to predict that the performance of a constant-BW optical system operating at relatively (with respect to the system BW) low bit rates will be better when retrieving the PAM-M signal (sampled at point A) than when retrieving the DB-PAM-M signal (sampled at point B). Under these circumstances, the eye-opening height and width of the "traditional" eyes become larger because there are fewer amplitude levels involved. However, for relatively high-speed transmission rates (or, equivalently, under demanding opto-electronic BW conditions), better performance is expected from retrieving the DB-PAM-M signal. This occurs because although a shorter eye-opening height and a narrower width will be measured from the corresponding eyes, these parameters become, in the DB case, more tolerant to the increase in bit rate (or overall system BW reduction), as already shown through simulations.
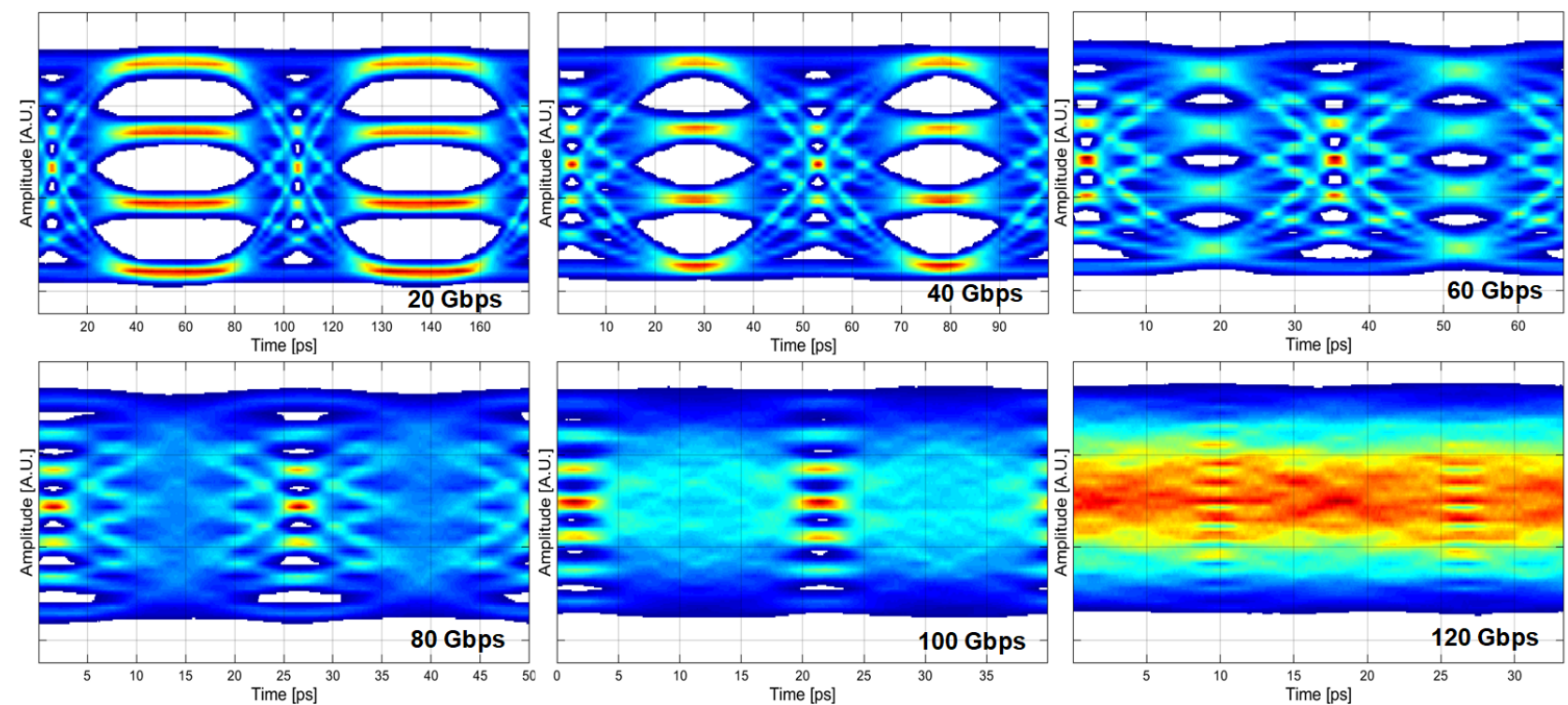

Figure 5. Received eye diagrams of PAM-4 signal for different bit rates when the high BW system (effective receiver BW of $23 \mathrm{GHz}$ ) in BtB configuration is simulated.

\section{Optimum Modulation Format: Transition Points}

\subsection{Back-to-Back Case}

To quantitatively demonstrate our observations through experiments, we measured the BER of the received signal for different bit rate values. In these measurements, the minimum BER that we can report with confidence, limited by the number of bits employed for the error-counting calculation, is $10^{-6}$. Figure 6a shows the results for the low-BW system (described in Section 3) in BtB configuration. Pre-coded PAM-2 and PAM-4 signals are launched, whereas PAM-M and DB-PAM-M signals, $M=2,4$, are retrieved from sampling points $A$ and B, respectively, as described in Sections 2 and 3. It can indeed be observed that, for bit rates lower than $24 \mathrm{~Gb} / \mathrm{s}$, PAM-2 shows a better BER performance. However, it is its DB counterpart that offers better performance for bit rates from 25 up to about $35 \mathrm{~Gb} / \mathrm{s}$. In the case of $M=4$, higher data rates can be reached. Initially, PAM-4 performs 
better, but at around $45 \mathrm{~Gb} / \mathrm{s}$ we observe a handover of the lead to DB-PAM-4, showing the very good performance of the latter up to approximately $60 \mathrm{~Gb} / \mathrm{s}$, where excessively high values of BER (above $10^{-2}$ ) are measured. Figure 6a confirms the existence of the transition points, found at $24 \mathrm{~Gb} / \mathrm{s}$ (for PAM-2/DB-PAM-2) and $45 \mathrm{~Gb} / \mathrm{s}$ (for PAM-4/DB-PAM-4). They have also recently been observed in a similar performance analysis [25]. Figure 6a also shows that, concerning performance, $M=4$ should generally be preferred over $M=2$ in systems that involve bit rates beyond $25 \mathrm{~Gb} / \mathrm{s}$. This bound, however, varies according to the system BW conditions, as shown in Figure 6b. The performance comparison of non-DB PAM-M modulation formats have been discussed in [5].

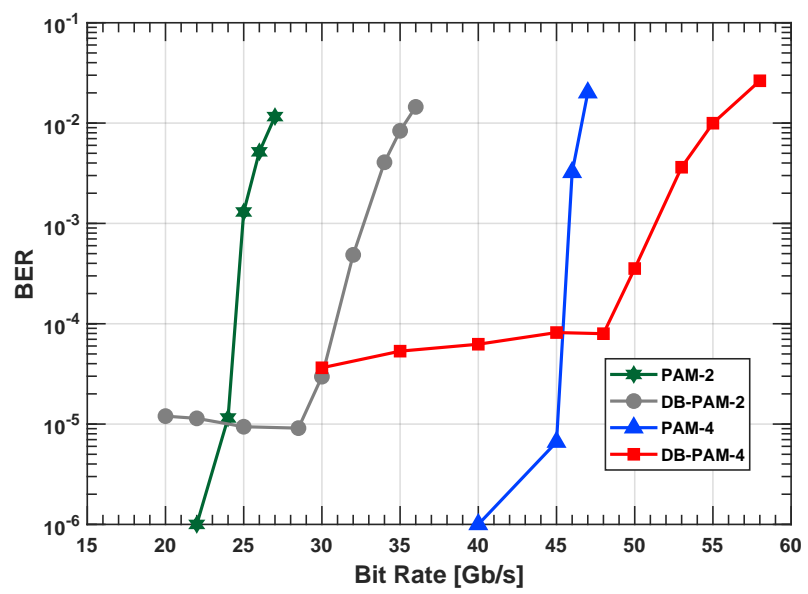

(a)

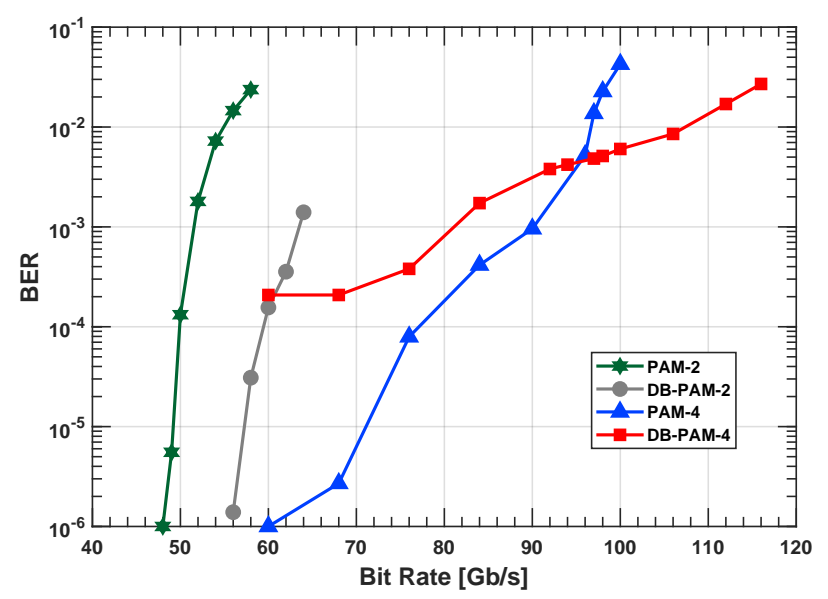

(b)

Figure 6. Measured BER as a function of bit rate for the (a) low BW (3-dB receiver BW of 7.3 GHz) and (b) high BW (3-dB receiver $\mathrm{BW}$ of $15.3 \mathrm{GHz}$ ) systems in BtB configuration using PAM-2,4 and DB-PAM-2,4 modulation formats.

We also carried out experiments with the high BW system operated in BtB. Figure $6 \mathrm{~b}$ shows the resulting BER versus bit rate curves. Similar behavior to that shown in Figure 6a is observed, although higher bit rates can now be reached. Again, the DB-PAM-2 modulation format performs better than PAM-2 at a higher speed. The transition (or crossing) point here occurs at a very low BER and, therefore, is not discernible due to the limited number of bits employed for the error-counting calculations (i.e., we cannot reliably calculate BER values below $10^{-6}$ ). In contrast with the $M=2$ case, the transition point is evident for $M=4$. The transition of performance between PAM- 4 and DB-PAM-4 occurs at about $96 \mathrm{~Gb} / \mathrm{s}$. Beyond that point, the use of DB-PAM-4 results in fewer transmission errors, whereas the opposite occurs at lower bit rates. Moreover, when DB-PAM-4 is employed together with forward-error correction (FEC), so-called "error-free" transmission at $106 \mathrm{~Gb} / \mathrm{s}$ is feasible for a BER of $10^{-2}$, corresponding with the low-density parity-check (LDPC) FEC threshold [38].

Figure 7 shows the measured eye diagrams for bit rate values around the transition point of $96 \mathrm{~Gb} / \mathrm{s}$, that is, where, according to Figure $6 \mathrm{~b}$, the measured BER for both $M=4$ modulation formats is practically the same. The closure of the eyes as the bit rate grows in the PAM-4 case agrees with the fast increase in the BER values observed from the blue curve with triangles in Figure $6 \mathrm{~b}$. At $100 \mathrm{~Gb} / \mathrm{s}$, the four levels cannot be differentiated. In the DB-PAM-4 case, this effect is less severe, leading to a (red with squares) curve having a lower slope. Yet, at $100 \mathrm{~Gb} / \mathrm{s}$, the central eyes practically look closed, but the seven levels can still be distinguished. The BER floor that is exhibited in Figure $6 \mathrm{a}, \mathrm{b}$ by the DB-PAM-2,4 curves at a low bit rate can be explained from the phenomenon already discussed at the beginning of Section 4. Indeed, according to Figure 5, the eye-opening of the six eyes (corresponding to DB transmission) remains practically unaffected during a considerable bit rate interval, thus leading to a constant BER value. This behavior changes at a very 
low bit rate (or equivalently, very ample system BW conditions) because, under these circumstances, the opening of these eyes starts degrading, leading to an eventual increase in BER. Conversely, the effect of reducing the bit rate on the three eyes corresponding to "traditional" (non-DB) transmission results in very clean, undistorted eyes, and hence excellent system performance. Under these very favorable transmission conditions, the use of DB-PAM-M modulation no longer makes sense.
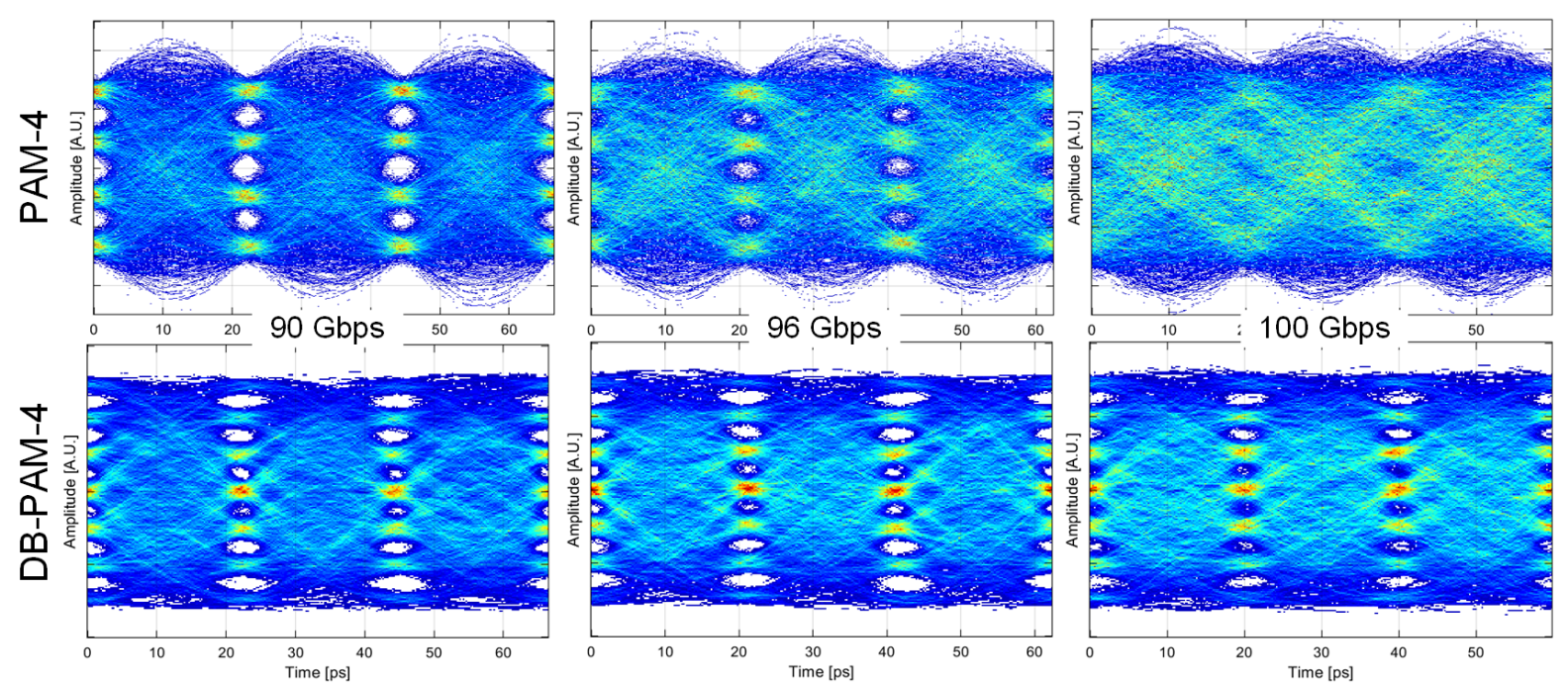

Figure 7. Received electrical eye diagrams for PAM-4 and DB-PAM-4 measured in the high BW system in BtB configuration. The corresponding BER values are graphed in Figure $6 \mathrm{~b}$. At $96 \mathrm{~Gb} / \mathrm{s}$, both formats share approximately the same BER value and represent the transition point.

Figure 6 can also be used to calculate, for a given maximum BER threshold, how much gain is achieved in terms of bit rate by switching from one modulation format to another. For the low- and high-BW systems, Table 2 presents the maximum speed that each format can reach, considering an upper bound for the BER of $10^{-2}$; this limit is commonly employed in connection to LDPC FEC [38]. For the low-BW scenario, the maximum transmission speed that can be reached employing PAM-2 is slightly greater than $25 \mathrm{~Gb} / \mathrm{s}$. By switching the format to DB-PAM-2, this figure improves by $8.5 \mathrm{~Gb} / \mathrm{s}$. The difference increases further (an extra $11.4 \mathrm{~Gb} / \mathrm{s}$ ) when shifting to PAM-4. The maximum bit rate of $55 \mathrm{~Gb} / \mathrm{s}$ is attained only when using DB-PAM-4 modulation. This bit rate is $8.3 \mathrm{~Gb} / \mathrm{s}$ faster than the $46.7 \mathrm{~Gb} / \mathrm{s}$ exhibited by PAM-4. Similar results arise when considering the high-BW scenario. There, the maximum bit rate realized when using PAM- 2 is $55 \mathrm{~Gb} / \mathrm{s}$. A $12 \mathrm{~Gb} / \mathrm{s}$ increase results from shifting this modulation format to its DB counterpart. A considerable growth of almost $30 \mathrm{~Gb} / \mathrm{s}$ is gained when using PAM-4 instead, leading to a maximum speed of $96.8 \mathrm{~Gb} / \mathrm{s}$. Switching to DB-PAM-4 represents a $10.2 \mathrm{~Gb} / \mathrm{s}$ increment; this results in a maximum bit rate of $107 \mathrm{~Gb} / \mathrm{s}$. Based on these numbers, one might conclude that DB-PAM-4 should be the modulation format of choice for the implementation of a practical transceiver, since it is able to reach the highest bit rate and produce an acceptable performance for a wide range of bit rate values. Nevertheless, different conclusions might be drawn when setting different values for the BER threshold. This is a consequence of the existence of the previously discussed transition points. Indeed, let us assume, for instance, that a maximum BER of $10^{-4}$ is now considered for the high-BW scenario. Then, DB-PAM-4 will no longer be the format of choice. Instead, PAM-4 will produce better results, and, according to Figure $6 \mathrm{~b}$, the maximum transmission speed will decrease to $76 \mathrm{~Gb} / \mathrm{s}$. If, for some reason, DB-PAM-2 is selected in lieu of PAM-4, the maximum bit rate will fall $17 \mathrm{~Gb} / \mathrm{s}$ shorter. As this discussion demonstrates, the choice of optimum modulation format is, in general, not straightforward. This occurs because the system performance improvement that can be gained through adequately selecting the best modulation format is 
very dependent on the prevailing conditions of system bandwidth, bit rate, fiber dispersion, signal processing choice, performance limits and so on.

Table 2. Maximum bit rate [Gb/s] achieved by each format for $\mathrm{BER} \leq 10^{-2}$ in BtB configuration.

\begin{tabular}{lcccc}
\hline & PAM-2 & DB-PAM-2 & PAM-4 & DB-PAM-4 \\
\hline Low-BW & 26.8 & 35.3 & 46.7 & 55.0 \\
High-BW & 55.0 & $67.0($ EST) & 96.8 & 107.0 \\
\hline
\end{tabular}

EST: estimated.

It is evident from the measurements presented in Figure $6 a, b$ that the transition point (bit rate) where the DB format surpasses in terms of BER performance its PAM-2,4 counterpart is very dependent on the overall system BW conditions. Therefore, the optimum choice of modulation format for a given system and bit rate has to be determined on a case-by-case basis, either experimentally or through simulations. Notwithstanding, we can always say that, in general, PAM-2,4 formats should be preferred when ample BW system conditions prevail and relatively low bit rates are involved. In contrast, DB-PAM-2,4 should be the format of choice when transmitting in BW-constrained systems with relatively high data rates. Moreover, as is well-known, higher cardinality formats are, in general, better suited for high-speed transmission [5], because they transmit a higher amount $\left(\log _{2}(M)\right)$ of bits per symbol. Note, however, that $M$ is also limited by the SNR of the system, since the use of a large number of amplitude levels results in indistinguishable eyes in noisy environments. Further research in this domain is, therefore, welcome [36,39].

\subsection{The Effect of Fiber Dispersion}

The choice of the optimum modulation format to increase the system throughput or reduce the BER for certain bit rates is also determined by the CD produced by the transmission fiber. Consequently, the determination of the transition points for a given system is also influenced by the amount of fiber $\mathrm{CD}$. To gain some insight into the context of O-band, we carried out $10-\mathrm{km}$ reach propagation experiments over SSMF, leading to BER versus bit rate curves practically identical to the ones shown in Figure 6a (consequently, these are not displayed). This occurs because the approximate accumulated CD, in this case, is only $-6 \mathrm{ps} / \mathrm{nm}$. It, therefore, has a very low impact on system performance.

To extend the analysis beyond our experimental capabilities, we carried out simulations using the computational infrastructure described in Section 3. Figure 8 shows calculations of dispersion penalty for the LDPC-FEC threshold of BER $=10^{-2}$ [38], as the fiber-accumulated CD is varied for three of the modulation formats investigated in this work. We simulated the high BW system operating at $60 \mathrm{~Gb} / \mathrm{s}$. We chose this bit rate because it results in a similar performance for the three analyzed modulation formats. The bit rate was too high to include PAM-2 in the numerical analysis. The figure shows that, at this medium-range bit rate, DB-PAM-4 tolerates CD very well, presenting a $1.4 \mathrm{~dB}$ penalty only after having reached $+200 \mathrm{ps} / \mathrm{nm}$. This means that, under the aforementioned conditions, DB-PAM-4 is useful for 10-km SSMF transmission (or slightly longer fiber length) in C-band. This can be confirmed in Figure 9a, which shows the dispersion penalty presented in Figure 8 , but this time displayed as a function of fiber length for particular values of wavelength $(\lambda=1550 \mathrm{~nm})$ and CD (18 ps $/ \mathrm{nm}-\mathrm{km})$. Although somewhat more pronounced, Figure 8 shows that a similar increase in penalty is observed for fiber propagation that exhibits negative CD. The situation is different with PAM-4, whose penalty markedly increases as CD grows, irrespective of the CD sign. The performance of this modulation format quickly deteriorates under the presence of $C D$, except for an interval running from -90 to $0 \mathrm{ps} / \mathrm{nm}$, where a negative power penalty (explained below) is observed. As shown in Figure 9a, the performance of this modulation format in C-band is rather poor, barely reaching 3-km transmission at a 2-dB dispersion penalty limit. The tolerance to CD of DB-PAM-2 can be considered rather moderate. According to Figure 8, DB-PAM-2 stands between PAM-4 and DB-PAM-4 for most of the investigated dispersion values. For the analyzed circumstances, 
this format presents a penalty of $2 \mathrm{~dB}$ at $-145 \mathrm{ps} / \mathrm{nm}$ and $85 \mathrm{ps} / \mathrm{nm}$. The latter corresponds to about $4.5 \mathrm{~km}$ propagation in C-band when employing an SSMF, as shown in Figure 9 a. The use of longer fiber lengths leads to unacceptably higher penalties. In O-band, close to the ZDW, the CD tolerance of DB-PAM-2 is similar to that of the other analyzed formats, especially when propagating the waveform over fiber with negative dispersion. For instance, for $\mathrm{D}=-0.6 \mathrm{ps} / \mathrm{nm}-\mathrm{km}$, corresponding to the wavelength used in our experiments $(\lambda=1310 \mathrm{~nm})$, the dispersion penalty exhibited by all analyzed formats is practically immaterial up to almost $200 \mathrm{~km}$; see Figure $9 \mathrm{~b}$. A discrepancy of DB-PAM-2 and, especially, PAM-4 with respect to DB-PAM-4, however, starts to grow as we increase the fiber length and/or move away from the ZDW; that is, when the absolute value of the accumulated dispersion increases. According to Figure $9 \mathrm{~b}$, the discrepancy at $290 \mathrm{~km}$ amounts to $3 \mathrm{~dB}$ for DB-PAM-2 and more than $7 \mathrm{~dB}$ for PAM-4. Hence, concerning CD tolerance, the use of DB-PAM-4 represents, in general, the best choice. The results presented in Figures 8 and 9, which demonstrate the prominence of DB-PAM-4 in terms of insensitivity to CD, may, in part, be understood by remembering that an increase in accumulated dispersion can be interpreted as a narrowing of the fiber BW, and consequently, of the overall system BW [40]. Since, in general, partial-response signaling performs better than standard (non-DB) modulation formats under relatively constrained bandwidth conditions (see Section 4), then DB-PAM-2, and, in particular DB-PAM-4, are expected to withstand the deleterious effect of $\mathrm{CD}$ across a greater interval of increasing accumulated dispersion than their non-DB counterparts. This is precisely what Figures 8 and 9 demonstrate.

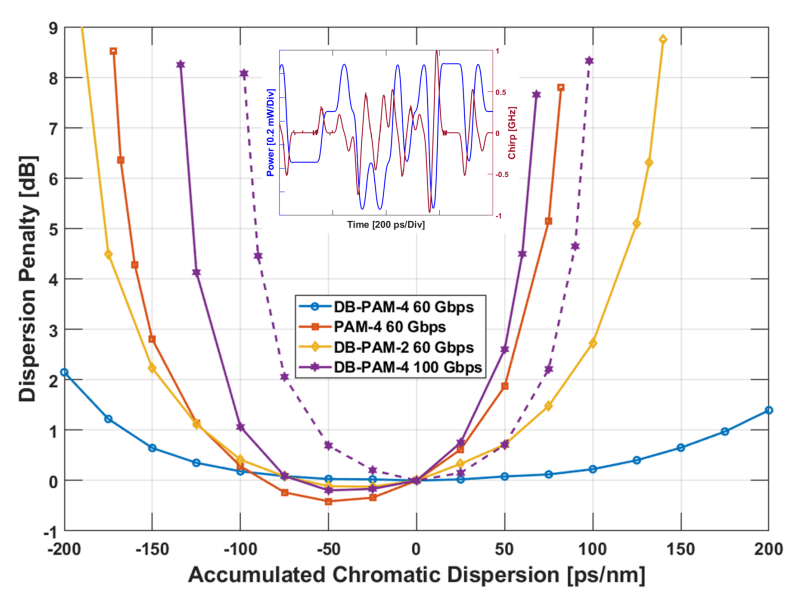

Figure 8. Simulated dispersion penalty as a function of accumulated fiber chromatic dispersion for different modulation formats and bit rates of 60 and $100 \mathrm{~Gb} / \mathrm{s}$ employing the high BW system. The dashed line corresponds to the DB-PAM- $4100 \mathrm{~Gb} / \mathrm{s}$ signal produced with a zero-frequency chirp transmitter. The inset shows the optical power (blue) of an $800 \mathrm{ps}$ segment of the $60 \mathrm{~Gb} / \mathrm{s}$ signal and its associated chirp (red) calculated just after the modulator. 


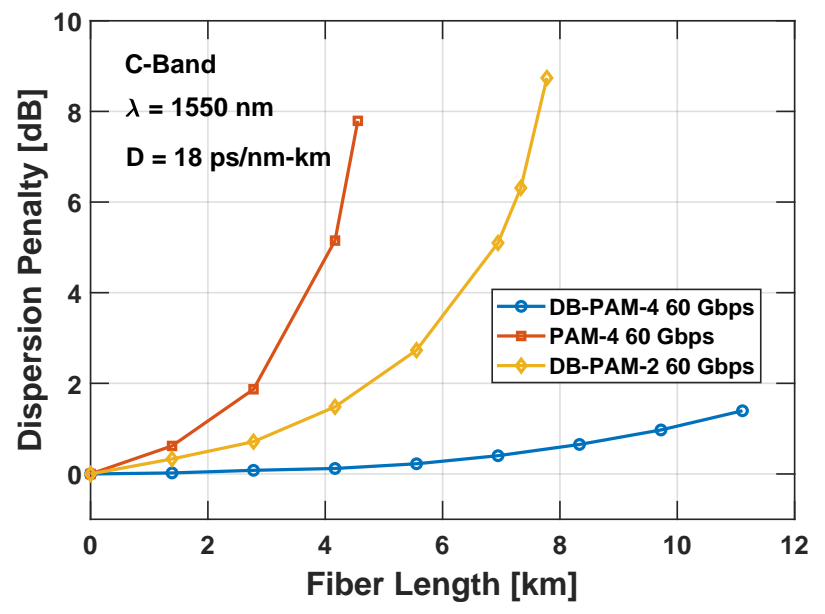

(a)

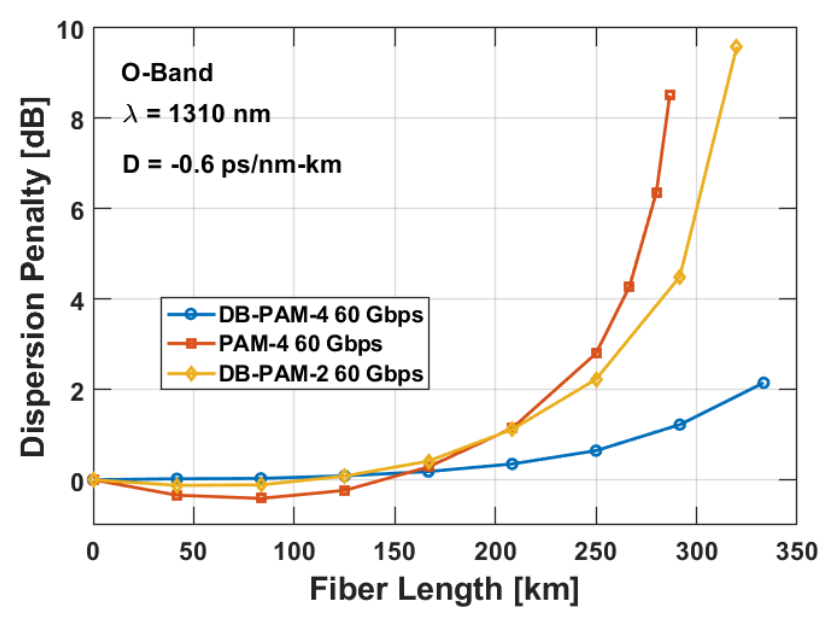

(b)

Figure 9. Simulated dispersion penalty as a function of fiber length for different modulation formats employing the high BW system. Transmission in (a) C-band and (b) O-band is considered.

It is also interesting to note that Figure 8 displays an asymmetry of the power penalty curves with respect to the zero-dispersion point. Some formats even exhibit a negative power penalty at around $-50 \mathrm{ps} / \mathrm{nm}$. This is explained by the interaction of the optical transmitter chirp with the negative CD. Indeed, as is well-known [41], the propagation of a signal with negative chirp over a negative-dispersion fiber of adequate length impacts favorably on system performance via pulse compression. The opposite occurs when a positive-dispersion fiber is employed. The inset in Figure 8, which presents a short interval of the power and corresponding frequency chirp of the $60 \mathrm{~Gb} / \mathrm{s}$ PAM-4 signal prior to entering the fiber, confirms a blue- (red-) shift in the frequency components in the pulse's leading (trailing) edge, corresponding to negative chirping. Since the simulated MZM is operated in push-pull mode, the amount of chirp produced by the modulator is relatively low: $2 \mathrm{GHz}$ of maximum excursion.

If we now analyze the behavior of a signal operating at a higher bit rate of $100 \mathrm{~Gb} / \mathrm{s}$, it can be anticipated that it will become drastically affected by $C D$, irrespective of the modulation format. Even DB-PAM-4, whose dispersion penalty is shown using a solid line with stars in Figure 8, exceeds the penalty produced by PAM-4 at $60 \mathrm{~Gb} / \mathrm{s}$ (orange line with squares). Despite this observation, and per similar comparative analyses [25], DB-PAM-4 remains the format of choice for high-speed transmission. Care has to be taken, however, to keep the difference between the selected transmission wavelength and the ZDW of the fiber within reasonable limits; otherwise, dispersion compensation becomes utterly necessary. At a high data rate, propagation in O-band instead of C-band should hence be preferred, as shown by our results. Figure 8 also displays, in a dashed line, the power penalty obtained with the DB-PAM-4 $100 \mathrm{~Gb} / \mathrm{s}$ signal (similar to the magenta solid line with stars), but this time produced with an MZM exhibiting a zero $\alpha$-factor. The even symmetry of the dashed curve around $0 \mathrm{ps} / \mathrm{nm}$ confirms that negative chirping is indeed responsible for the shift towards negative dispersion values which is observed in the rest of the (solid) curves. This analysis shows that the amount of chirp imprinted by the transmitter in the signal is, therefore, another parameter that must be taken into account when calculating the position of the transition points already discussed. In general, negative (positive) dispersion fibers favor the use of negatively (positively) chirped transmitters [42].

Finally, it must be mentioned that we have numerically investigated the effect of pulsebroadening induced by polarization-mode dispersion (PMD) in the $60 \mathrm{~Gb} / \mathrm{s}$ analyzed signals. For that purpose, we calculated the variation in BER for optical fibers up to $100 \mathrm{~km}$ long and exhibiting a PMD parameter $D_{p} \leq 0.5 \mathrm{ps} / \sqrt{\mathrm{km}}$. We considered the highbandwidth system described in Section 3 with a marginal fiber dispersion of $1 \mathrm{ps} / \mathrm{nm}-\mathrm{km}$ 
and zero attenuation. We found that, under these circumstances, the BER variation as a function of $D_{p}$ is negligible, as expected from a short-reach IM/DD system deployed with currently available commercial optical fibers.

\section{Hypothetical Format-Selective Transceiver}

Our experimental and numerical analysis demonstrates that the optimum choice of modulation format depends on the overall BW, data rate, phase-modulation characteristics of the transmitter, and the fiber CD specifications of the particular photonic system. Moreover, it serves as an aid to make this choice. However, our investigation also points out the convenience of employing a format-selective transceiver in multi-rate optical networks utilizing opto-electronic BW-constrained links. Indeed, such an adaptive multi-format transceiver would be able to maintain the system performance tolerances within reasonable limits in case the data rate, CD, or BW conditions of the link eventually change [43] — for example, in response to sudden network traffic demand variations, a link length increase, or to guarantee the interoperability of components belonging to a myriad of vendors that exhibit different BW specifications. For that, calculation of the aforementioned defined transition points for the particular link would be necessary. However, once computed, switching back and forth between the PAM and DB-PAM formats would be carried out by simply changing (even at a software level) the sampling point and slicing levels in the receiver. For instance, let us assume SSMF propagation close to the ZDW along $10 \mathrm{~km}$ in a photonic system having $10 \mathrm{G}$ components. According to Figure 6a, the use of a formatselective receiver operating at sampling point A (see Figure 1), allows the transmission of a PAM-4 signal running at $20 \mathrm{GBd}$ with a BER below $10^{-3}$. However, if a data rate upgrade to $25 \mathrm{GBd}$ is required, the same receiver will have to switch the modulation format to DB-PAM-4 because this symbol rate lies beyond the transition point. The receiver now operates at sampling point B and increases the threshold count, thus maintaining the required BER performance level. Selection of the best modulation format can also be carried out automatically aided by a control algorithm that optimizes a performance metric (e.g., the BER). For that, the implementation of a low-rate feedback channel from the receiver to transmitter, together with the use of pilot symbols for setup, might be convenient. Note that although the same receiver is employed in the PAM-4 and DB-PAM-4 cases, different parameter configurations for sampling (A/B), thresholding (3/6 levels), and signal processing (FFE) will be required in each case. As mentioned before, depending on the actual transceiver implementation, this can be performed at a software level, as we did in our experiments. Moreover, the proposed transmitter may simply be built on the already existing transceiver infrastructure available for PAM-4 modulation, thus increasing the feasibility of the proposed solution. When transmission away from the ZDW is required, the effect of residual fiber dispersion must be taken into account. In this case, the chirp induced by the transmitter in the optical waveform acts as an extra degree of freedom to optimize the position of the transition points for the application of interest. Indeed, by properly adjusting the operating point of the particular transmitter (e.g., by varying the driving voltage and current), fine-tuning the effective chirp parameter, and the associated interaction of the instantaneous optical frequency with the fiber CD, optimum performance can be achieved [44]. This, in turn, allows control of the overall system performance and, hence, of the location of the transition points in the BER versus bit rate plane.

It is important to note that the implementation of the format-selective transmitter strongly relies on the existence of the aforementioned transition points, uncovered through our analysis. As long as these points exist, the format-selective transceiver can employ other kinds of signaling. For instance, although based on a more involved implementation, attractive options are the recently proposed Tomlinson-Harashima precoded PAM-M optical modulation formats, whose optimum choice is also determined by the system conditions and bit rate $[45,46]$. 


\section{Conclusions}

We have investigated the performance of PAM-2 and PAM-4, but more importantly, their duobinary counterparts, in two photonic systems exhibiting different opto-electronic BW conditions. Our experimental and numerical analyses show that the optimum choice of format depends on the system's BW, chromatic dispersion, optical chirp, and desired throughput, and it is determined by the so-called transition points for a particular transmission system. These points refer to the optimum bit rate values at which a transition from one modulation format to another is appropriate to maintain certain system performance. In general terms, duobinary formats should only be employed in BW-constrained environments where high transmission speeds are involved, for instance, in optical links with transceivers with low opto-electronic BW with respect to the data rate. Due to their tolerance to the effect of fiber chromatic dispersion, duobinary formats should also be preferred in the presence of a conspicuous level of this deleterious effect. Here, we experimentally showed that, by using DB-PAM-4, back-to-back transmission at a bit rate of $106 \mathrm{~Gb} / \mathrm{s}$ can be achieved over a $25 \mathrm{G}$ environment employing minimum DSP. Naturally, an increase in power penalty is expected as dispersion accumulates over the link. PAM-M is preferable under low demanding bandwidth conditions. These conclusions, together with the fact that these IM/DD formats share similar implementation details, naturally led to the proposal of a (DB)-PAM-based format-selective transceiver, here discussed for the first time. Apart from its relative simplicity, its main feature is that using components developed for $25 \mathrm{~Gb} / \mathrm{s}$ systems allows transmission at different distances and bit rates, from around 20 to $100 \mathrm{~Gb} / \mathrm{s}$, without introducing an unnecessary performance penalty. The link capacity can, therefore, be dynamically adjusted depending on the propagation condition or any network reconfiguration. This will make possible the use of a single transceiver over a wide range of applications, thus increasing its meaning. The proposed adaptive transceiver also leverages the use of multi-level modulation formats in combination with their doubinary versions in IM/DD systems. We hope that the ideas treated in this article will help in the design of next-generation photonic systems for 5G, 100G PON, and $800 \mathrm{GbE}$ networks.

Author Contributions: Conceptualization, R.G.-C., M.G.S., D.E.C.-H., F.C., L.G., R.B., D.V.P.; Investigation, R.G.-C., M.G.S., M.S.A., Z.X., E.E.-F., G.V.-L., L.G., R.B.; Software, R.G.-C, M.G.S., Z.X.; Supervision, F.C., S.L., D.V.P.; Writing, All. All authors have read and agreed to the published version of the manuscript.

Funding: The work of M.G.S. was supported in part by the Fonds de Recherche du Québec-Nature et technologies (FRQNT) under Grant 200755, in part by the RH Tomlinson Fellowship of McGill University under Grant 90025, and in part by the SPIE Optics and Photonics Education Scholarship (2019). R.G.-C. is grateful to Dirección General de Asuntos del Personal Académico, UNAM for PAPIIT grant IN103720.

Institutional Review Board Statement: Not applicable.

Informed Consent Statement: Not applicable.

Data Availability Statement: The data that supports the findings of this study are available from the corresponding author upon reasonable request.

Acknowledgments: We are indebted to B. Snow for his computational infrastructure support.

Conflicts of Interest: The authors declare no conflict of interest. 


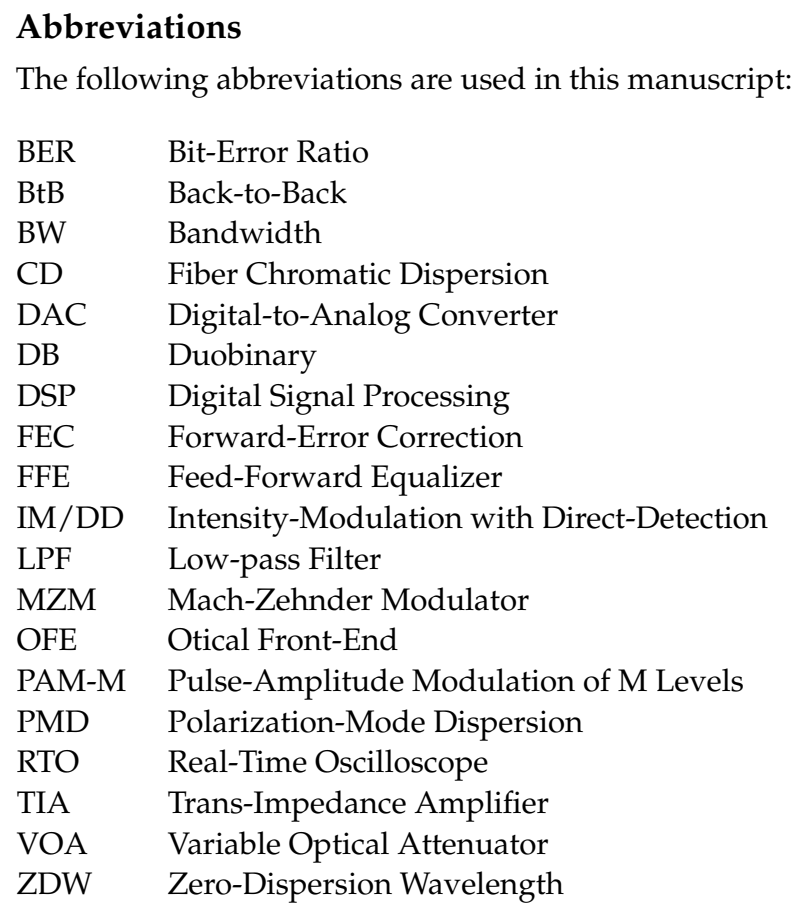

\section{References}

1. Zhong, K.; Zhou, X.; Gui, T.; Tao, L.; Gao, Y.; Chen, W.; Man, J.; Zeng, L.; Lau, A.P.T.; Lu, C. Experimental study of PAM-4, CAP-16, and DMT for $100 \mathrm{~Gb} / \mathrm{s}$ short reach optical transmission systems. Opt. Express 2015, 23, 1176-1189. [CrossRef] [PubMed]

2. Xu, X.; Zhou, E.; Liu, G.N.; Zuo, T.; Zhong, Q.; Zhang, L.; Bao, Y.; Zhang, X.; Li, J.; Li, Z. Advanced modulation formats for 400-Gbps short-reach optical inter-connection. Opt. Express 2015, 23, 492-500. [CrossRef] [PubMed]

3. Saber, M.G.; Morsy-Osman, M.; Hui, M.; El-Fiky, E.; Vall-Llosera, G.; Dortschy, B.; Urban, P.J.; Cavaliere, F.; Lessard, S.; Plant, D.V. DSP-free 25 Gbit/s PAM-4 Transmission using 10G Transmitter and Coherent Amplification. IEEE Photonics Technol. Lett. 2018, 30, 1547-1550. [CrossRef]

4. Saber, M.G.; El-Fiky, E.; Xing, Z.; Morsy-Osman, M.; Patel, D.; Samani, A.; Alam, M.S.; Shahriar, K.A.; Xu, L.; Vall-Llosera, G.; et al. 25 and $50 \mathrm{~Gb} / \mathrm{s} / \lambda$ PAM-4 Transmission Over 43 and 21 km Using a Simplified Coherent Receiver on SOI. IEEE Photonics Technol. Lett. 2019, 31, 799-802. [CrossRef]

5. Chagnon, M.; Osman, M.; Poulin, M.; Latrasse, C.; Gagné, J.F.; Painchaud, Y.; Paquet, C.; Lessard, S.; Plant, D. Experimental study of $112 \mathrm{~Gb} / \mathrm{s}$ short reach transmission employing PAM formats and SiP intensity modulator at $1.3 \mu \mathrm{m}$. Opt. Express 2014, 22, 21018-21036. [CrossRef]

6. IEEE P802.3bs 400Gb/s Ethernet Task Force. 2017. Available online: http:/ /www.ieee802.org/3/bs/ (accessed on 1 March 2021).

7. Harstead, E.; Van Veen, D.; Houtsma, V.; Dom, P. Technology Roadmap for Time-Division Multiplexed Passive Optical Networks (TDM PONs). J. Light. Technol. 2019, 37, 657-664. [CrossRef]

8. Saber, M.G.; Vall-Llosera, G.; Patel, D.; Samani, A.; Li, R.; Morsy-Osman, M.; Chagnon, M.; El-Fiky, E.; Gutiérrez-Castrejón, R.; Urban, P.J.; et al. Silicon-based optical links using novel direct detection, coherent detection and dual polarization methods for new generation transport architectures. Opt. Commun. 2019, 450, 48-60. [CrossRef]

9. Winzer, P.J.; Essiambre, R.J. Advanced modulation formats for high-capacity optical transport networks. Proc. IEEE 2006, 49, 952-985. [CrossRef]

10. Frejstrup Suhr, L.; Vegas Olmos, J.J.; Mao, B.; Xu, X.; Liu, G.N.; Tafur Monroy, I. Direct modulation of 56 Gbps duobinary-4-PAM. In Proceedings of the Optical Fiber Communications Conference and Exhibition (OFC), Los Angeles, CA, USA, 22-26 March 2015; p. 1-3.

11. Frejstrup Suhr, L.; Madsen, P.; Tafur Monroy, I.; Vegas Olmos, J.J. Analog-based duobinary-4-PAM for electrical bandwidth limited optical fiber links. Opt. Appl. 2016, XLVI, 71-78. [CrossRef]

12. Yang, C.; Hu, R.; Luo, M.; Yang, Q.; Li, C.; Li, H.; Yu, S. IM/DD-Based 112-Gb/s/lambda PAM-4 Transmission Using 18-Gbps DML. IEEE Photonics J. 2016, 8, 1-7. [CrossRef]

13. Zhang, Q.; Stojanovic, N.; Prodaniuc, C.; Xie, C.; Koenigsmann, M.; Laskowski, P. Single-lane 180 Gbit/s PAM-4 signal transmission over $2 \mathrm{~km}$ SSMF for short-reach applications. Opt. Lett. 2016, 41, 4449-4452. [CrossRef]

14. Dong, J.; Hu, R. Transmission of 112 (4x28)-Gb/s PAM-4 signal over 48.6-km SSMF within only 50-GHz grid. Opt. Commun. 2016, 381, 200-204. [CrossRef]

15. Saber, M.G.; Gutiérrez Castrejón, R.; Alam, M.S.; Xing, Z.; El-Fiky, E.; Xu, L.; Cavaliere, F.; Vall-Llosera, G.; Lessard, S.; Plant, D.V. $100 \mathrm{~Gb} / \mathrm{s} / \lambda$ duo-binary PAM-4 transmission using 25G components achieving $50 \mathrm{~km}$ reach. IEEE Photonics Technol. Lett. 2019, 32, 138-141. [CrossRef] 
16. Walklin, S.; Conradi, J. Multilevel signaling for increasing the reach of $10 \mathrm{~Gb} / \mathrm{s}$ lightwave systems. J. Light. Technol. 1999, 17, 2235-2248. [CrossRef]

17. Lender, A. Correlative digital communication techniques. IEEE Trans. Commun. Technol. 1964, 12, 128-135. [CrossRef]

18. Zhang, Q.; Stojanovic, N.; Zuo, T.; Zhang, L.; Prodaniuc, C.; Karinou, F.; Xie, C.; Zhou, E. Single-lane 180 Gb/s SSB-duobinaryPAM-4 signal transmission over $13 \mathrm{~km}$ SSMF. In Proceedings of the 2017 Optical Fiber Communications Conference and Exhibition (OFC) Technical Digest, Los Angeles, CA, USA, 19-23 March 2017; p. Tu2D.2.

19. Suhr, L.F.; Olmos, J.V.; Mao, B.; Xu, X.; Liu, G.; Monroy, I.T. 112-Gbit/s $\times$ 4-lane duobinary-4-PAM for 400GBase. In Proceedings of the 2014 The European Conference on Optical Communication (ECOC), Cannes, France, 21-25 September 2014; p. 1-3.

20. Zhang, Q.; Stojanovic, N.; Wei, J.; Xie, C. Single-lane 180 Gb/s DB-PAM-4-signal transmission over an 80 km DCF-free SSMF link. Opt. Lett. 2017, 42, 883-886. [CrossRef]

21. Zhang, K.; Zhuge, Q.; Xin, H.; Hu, W.; Plant, D.V. Performance comparison of DML, EML and MZM in dispersion-unmanaged short reach transmissions with digital signal processing. Opt. Express 2018, 26, 34288-34304. [CrossRef] [PubMed]

22. Madsen, P.; Frejstrup Suhr, L.; Rodriguez, J.S.; Tafur Monroy, I.; Vegas Olmos, J.J. Performance evaluation of multilevel modulation formats using partial response for capacity upgrade in access network with limited electronic bandwidth. Opt. Fiber Technol. 2016, 31, 168-171. [CrossRef]

23. Liu, G.N.; Zhang, L.; Zuo, T.; Zhang, Q. IM/DD Transmission Techniques for Emerging 5G Fronthaul, DCI, and Metro Applications. J. Light. Technol. 2018, 36, 560-567. [CrossRef]

24. Eiselt, N.; Muench, D.; Dochhan, A.; Griesser, H.; Eiselt, M.; Olmos, J.J.V.; Monroy, I.T.; Elbers, J. Performance Comparison of 112-Gb/s DMT, Nyquist PAM4, and Partial-Response PAM4 for Future 5G Ethernet-Based Fronthaul Architecture. J. Light. Technol. 2018, 36, 1807-1814. [CrossRef]

25. Torres-Ferrera, P.; Wang, H.; Ferrero, V.; Gaudino, R. 100 Gbps / $\lambda$ PON downstream O- and C-band alternatives using directdetection and linear-imparment equalization. IEEE/OSA J. Opt. Commun. Netw. 2021, 13, A111-A123. [CrossRef]

26. Saber, M.G.; Gutiérrez-Castrejón, R.; Xing, Z.; Alam, M.S.; El-Fiky, E.; Ceballos-Herrera, D.E.; Cavaliere, F.; Vall-Llosera, G.; Lessard, S.; Plant, D.V. Demonstration of $108 \mathrm{~Gb}$ /s duo-binary PAM-8 transmission and the probabilistic modeling of DB-PAM-M BER. IEEE Photonics J. 2021, 13, 1-14. [CrossRef]

27. Ohlendorf, S.; Rosenkranz, W. Flexible optical modulation technologies for data center applications. In Proceedings of the 2017 19th International Conference on Transparent Optical Networks (ICTON), Girona, Spain, 2-6 July 2017; pp. 1-4.

28. Loussouarn, Y.; Pincemin, E.; Pan, M.; Miller, G.; Gibbemeyer, A.; Mikkelsen, B. Multi-Rate Multi-Format CFP/CFP2 Digital Coherent Interfaces for Data Center Interconnects, Metro, and Long-Haul Optical Communications. J. Light. Technol. 2019, 37, 538-547. [CrossRef]

29. Bosco, G. Advanced Modulation Techniques for Flexible Optical Transceivers: The Rate/Reach Tradeoff. J. Light. Technol. 2019, 37, 36-49. [CrossRef]

30. Torres-Ferrera, P.; Gutiérrez-Castrejón, R.; Tomkos, I. Multi-format 800-1600 Gb/s coherent transceiver for inter-data centre interconnects over SMF. In Proceedings of the 19th International Conference on Transparent Optical Networks (ICTON), Girona, Spain, 2-6 July 2017; pp. 1-4.

31. Roberts, K. Flexible optical transceivers. In Next-Generation Optical Communication: Components, Sub-Systems, and Systems VII; Li, G., Zhou, X., Eds.; International Society for Optics and Photonics, SPIE: San Francisco, CA, USA, 2018; Volume 10561, pp. 1-13. [CrossRef]

32. Moreolo, M.S.; Fàbrega, J.M.; Nadal, L.; Martín, L. Optical Technology Options for Programmable S-BVT. In Proceedings of the 20th International Conference on Transparent Optical Networks (ICTON), Bucharest, Romania, 1-5 July 2018; pp. 1-4.

33. IEEE P802.3ca 50G-EPON Task Force. 2019. Available online: https:/ /www.ieee802.org/3/ca/ (accessed on 6 February 2021).

34. Zhao, J.; Huo, L.; Chan, C.K.; Chen, L.K.; Lin, C. Analytical investigation of optimization, performance bound, and chromatic dispersion tolerance of 4-amplitude-shifted-keying format. In Proceedings of the 2006 Optical Fiber Communication Conference, Anaheim, CA, USA, 5-10 March 2006; p. JThB15. [CrossRef]

35. Kumar, S.; Deen, M.J. Fiber Optic Communications: Fundamentals and Applications; John Wiley and Sons Ltd.: Chichester, UK, 2014; pp. 172-174.

36. Howson, R. An Analysis of the Capabilities of Polybinary Data Transmission. IEEE Trans. Commun. Technol. 1965, 13, 312-319. [CrossRef]

37. Optiwave. Available online: https:/ / optiwave.com/ (accessed on 10 March 2021).

38. Zhao, D. LDPC for 100G EPON. IEEE P802.3ca 50G-EPON Task Force Meeting. 2017. Available online: http://grouper.ieee.org/ groups/802/3/ca/public/meeting_archive/2017/05/zhao_3ca_1_0517.pdf (accessed on 20 January 2021).

39. Feher, K. Digital Communications, Microwave Applications; Prentice Hall: Englewood Cliffs, NJ, USA, 1981 ; pp. 61-63.

40. Agrawal, G.P. Fiber-Optic Communication Systems, 3rd ed.; John Wiley \& Sons, Inc.: Hoboken, NJ, USA, $2002 ;$ pp. 53-55.

41. Tomkos, I.; Chowdhury, D.; Conradi, J.; Culverhouse, D.; Ennser, K.; Giroux, C.; Hallock, B.; Kennedy, T.; Kruse, A.; Kumar, S.; et al. Demonstration of negative dispersion fibers for DWDM metropolitan area networks. IEEE J. Sel. Top. Quantum Electron. 2001, 7, 439-460. [CrossRef]

42. Filios, A.; Gutierrez-Castrejon, R.; Tomkos, I.; Hallock, B.; Vodhanel, R.; Coombe, A.; Yuen, W.; Moreland, R.; Garrett, B.; Duvall, C.; et al. Transmission performance of a $1.5-\mu \mathrm{m} 2.5-\mathrm{Gb} / \mathrm{s}$ directly modulated tunable VCSEL. IEEE Photonics Technol. Lett. 2003, 15, 599-601. [CrossRef] 
43. Tomkos, I.; Azodolmolky, S.; Solé-Pareta, J.; Careglio, D.; Palkopoulou, E. A tutorial on the flexible optical networking paradigm: State of the art, trends, and research challenges. Proc. IEEE 2014, 102, 1317-1337. [CrossRef]

44. Borkowski, R.; Schmuck, H.; Cerulo, G.; Provost, J.; Houtsma, V.; van Veen, D.; Harstead, E.; Mallecot, F.; Bonk, R. The Impact of Transmitter Chirp Parameter on the Power Penalty and Design of 50 Gbit/s TDM-PON. In Proceedings of the 2020 Optical Fiber Communications Conference and Exhibition (OFC), San Diego, CA, USA, 8-12 March 2020; p. Th1B.5.

45. Xiang, M.; Xing, Z.; El-Fiky, E.; Morsy-Osman, M.; Zhuge, Q.; Plant, D.V. Single-Lane 145 Gbit/s IM/DD Transmission With Faster-Than-Nyquist PAM4 Signaling. IEEE Photonics Technol. Lett. 2018, 30, 1238-1241. [CrossRef]

46. Hu, Q.; Chagnon, M.; Schuh, K.; Buchali, F.; Bülow, H. IM/DD Beyond Bandwidth Limitation for Data Center Optical Interconnects. J. Light. Technol. 2019, 37, 4940-4946. [CrossRef] 